\title{
Body weight, fat mass and metabolic complications during androgen deprivation therapy: should urologists recommend exercise and diet to help patients overcome toxicities?
}

\author{
Daniel A. Galvão $\mathbb{I}^{1,2} \cdot$ Dennis R. Taaffe $\mathbb{I}^{1,2} \cdot$ Robert U. Newton $\mathbb{D}^{1,2}$
}

Received: 22 February 2021 / Revised: 12 March 2021 / Accepted: 23 March 2021 / Published online: 12 April 2021

(c) The Author(s), under exclusive licence to Springer Nature Limited 2021

Comprehensive Lifestyle Improvement Program for Prostate Cancer (CLIPP) is associated with improvement in weight and components of metabolic syndrome in men on androgen deprivation therapy for prostate cancer. Algotar AM et al. Prostate Cancer and Prostatic Diseases (In Press, 2021).

Overweight and obese men with prostate cancer have a higher risk of disease recurrence, metastatic disease, prostate cancer-specific mortality, cardiovascular disease-specific mortality and all-cause mortality [1, 2]. Problematically, obesity is exacerbated in men with prostate cancer undergoing androgen deprivation therapy (ADT) due to significant body composition alterations occurring particularly in the first year of treatment [3]. These men on ADT gain fat mass, lose lean mass, and experience a series of adverse effects from therapy including hyperglycemia, decreased insulin sensitivity, altered lipoprotein profile, and increased incidence of metabolic syndrome [4]. Such unfavorable metabolic changes from testosterone suppression increase risk factors for cardiovascular disease and is likely to mediate the mechanisms underlying the higher frequency of cardiovascular events observed in epidemiological studies on prostate cancer patients undergoing this form of treatment [5]. This is of considerable concern to patients and clinicians, as surviving the comorbidities associated with ADT can become more problematic than the original cancer and patients might decline to use ADT and clinicians might be more hesitant to prescribe ADT. This is especially important for men with

Daniel A. Galvão

d.galvao@ecu.edu.au

1 Exercise Medicine Research Institute, Edith Cowan University, Joondalup, WA, Australia

2 School of Medical and Health Sciences, Edith Cowan University, Joondalup, WA, Australia long life expectancies who are likely to be undertaking ADT for prolonged periods of time.

About 10 years ago, we suggested "exercise to be the frontrunner for overcoming the newly discovered ADTassociated risks" [6], and since then numerous studies have been conducted addressing ADT-related toxicities and in particular body composition and risk factors for metabolic syndrome and cardiovascular diseases. Recently, we published a summary of this literature in two comprehensive systematic reviews and meta-analyses including 21 exercise studies and 1748 prostate cancer patients (mostly during or following ADT) $[7,8]$ reporting that supervised exercise in men with prostate cancer improves fat mass $(-1 \%$ in body fat and $-0.6 \mathrm{~kg}$ in fat mass), lean mass $(\sim+0.5 \mathrm{~kg}$ in whole body and appendicular lean mass), functional capacity (i.e., chair rise, $400 \mathrm{~m}$ test, $6 \mathrm{~m}$ fast walk, and stair climb tests), and fitness outcomes (i.e., VंO2peak and muscle strength), reduces fatigue and improves quality of life. However, these studies utilized supervised exercise programs in well controlled settings and as such the scalability of these interventions may be questioned when referral pathways to exercise professionals are not readily available or appropriate facilities accessible. Moreover, the reach of these interventions to the broader community of men with prostate cancer and, in particular, those in regional and rural areas may be limiting.

In this issue of Prostate Cancer and Prostatic Diseases, Algotar et al. [9] reported preliminary results of the Comprehensive Lifestyle Improvement Program for Prostate Cancer (CLIPP) on outcomes of feasibility and initial efficacy in reducing obesity and metabolic syndrome in 31 men on or previously exposed to ADT. The intervention consisted of a multi-component lifestyle modification program delivered weekly for 16 weeks by a trained health coach and included nutrition, physical activity, and supportive strategies to promote and maintain behavior modification. The two main objectives were to assist men: (1) achieve and 
maintain $7 \%$ weight loss from their starting weight, and (2) to achieve and maintain $150 \mathrm{~min}$ of moderate intensity physical activity each week. The authors reported impressive changes for total weight $(-6.8 \mathrm{~kg})$, waist $(-6.3$ $\mathrm{cm})$, systolic BP $(-10.7 \mathrm{~mm}$ of $\mathrm{Hg})$, diastolic BP $(-7.1 \mathrm{~mm}$ of $\mathrm{Hg})$, triglycerides $(-32.2 \mathrm{mg} / \mathrm{dl}), \mathrm{HDL}(+3.9 \mathrm{mg} / \mathrm{dl})$ and serum glucose $(+10.8 \mathrm{mg} / \mathrm{dl})$ with high retention to the program and adherence rates of 90.3 and $100 \%$, respectively. The authors are commended on their efforts to plan and nicely execute a study aimed to improve body weight and metabolic complications in men with prostate cancer in desperate need to counteract ADT-treatment toxicities. It is essential to note that additional data on dual $\mathrm{x}$-ray absorptiometry are yet to be reported and will be important in determining if lean tissue was preserved given the critical need to maintain muscle mass while intentionally losing total body weight.

In addition, advice to increase physical activity as part of weight loss programs is predominantly based on aerobic exercise as opposed to resistance or anabolic exercise and, as such, not as specific to preserve lean mass particularly when large reductions in total body weight are intended. Recently, we reported that in obese ADT-treated prostate cancer patients, a targeted supervised clinic-based exercise (high volume of $300 \mathrm{~min}$ of exercise weekly) and nutrition intervention (daily energy deficit $2100-4200 \mathrm{~kJ}$ and protein supplementation) resulted in a substantial reduction in fat mass $(-2.8 \mathrm{~kg})$ over 3 months with muscle mass preserved [10]. A core component of the program was the specific inclusion of resistance training and protein supplementation aimed at preserving lean mass during weight loss. In contrast, we identified a substantial decline of lean mass accompanying a weight loss program prior to robot assisted radical prostatectomy where only aerobic exercise and a very low-calorie diet $(\sim 3000-4000 \mathrm{~kJ})$ were implemented [11]. These contrasting findings demonstrate the importance of considering exercise mode specificity and components of such interventions that will likely dictate changes in the outcomes of interest.

Apart from the critical and well established physical and physiological changes accompanying ADT, the psychological impact of the disease and treatment remains a considerable burden. To this end, various modes of supervised exercise (resistance and aerobic) are reported to reduce psychological distress in men with prostate cancer on ADT [12] and such data can further support programs such as CLIPP that are likely to influence psychological health in these patients in addition to the reported improvements in metabolic profile and body weight outcomes.

Given the vast amount of research evidence accumulated in the past decade, there is a need to ensure that exercise and nutritional interventions are now used to assist urologists to help their patients overcome ADT-related toxicities. While there is still a requirement for further trials to expand on the current results, clinicians should act now to help their patients counteract body fat gains, muscle loss, and metabolic complications during ADT. The tools are readily available and they need to be used!

\section{Compliance with ethical standards}

Conflict of interest The authors declare no competing interests.

Publisher's note Springer Nature remains neutral with regard to jurisdictional claims in published maps and institutional affiliations.

\section{References}

1. Cao Y, Ma J. Body mass index, prostate cancer-specific mortality, and biochemical recurrence: a systematic review and metaanalysis. Cancer Prev Res (Philos). 2011;4:486-501.

2. Troeschel AN, Hartman TJ, Jacobs EJ, Stevens VL, Gansler T, Flanders WD, et al. Postdiagnosis Body Mass Index, Weight Change, and Mortality From Prostate Cancer, Cardiovascular Disease, and All Causes Among Survivors of Nonmetastatic Prostate Cancer. J Clin Oncol. 2020;38:2018-27.

3. Smith MR, Finkelstein JS, McGovern FJ, Zietman AL, Fallon MA, Schoenfeld DA, et al. Changes in body composition during androgen deprivation therapy for prostate cancer. J Clin Endocrinol Metab. 2002;87:599-603.

4. Braga-Basaria M, Dobs AS, Muller DC, Carducci MA, John M, Egan J, et al. Metabolic syndrome in men with prostate cancer undergoing long-term androgen-deprivation therapy. J Clin Oncol. 2006;24:3979-83.

5. Keating NL, O'Malley AJ, Smith MR. Diabetes and cardiovascular disease during androgen deprivation therapy for prostate cancer. J Clin Oncol. 2006;24:4448-56.

6. Galvao DA, Newton RU, Taaffe DR, Spry N. Can exercise ameliorate the increased risk of cardiovascular disease and diabetes associated with ADT? Nat Clin Pr Urol. 2008;5:306-7.

7. Lopez P, Taaffe DR, Newton RU, Buffart LM, Galvão DA. What is the minimal dose for resistance exercise effectiveness in prostate cancer patients? Systematic review and meta-analysis on patientreported outcomes. Prostate Cancer Prostatic Dis. 2020. https://doi. org/10.1038/s41391-020-00301-4. [Epub ahead of print].

8. Lopez P, Taaffe DR, Newton RU, Galvao DA. Resistance exercise dosage in men with prostate cancer: systematic review, meta-analysis, and meta-regression. Med Sci Sports Exerc. 2021;53:459-69.

9. Algotar AM, Hsu CH, Chow HH, Dougherty ST, Babiker HM, Marrero DG, et al. Comprehensive Lifestyle Improvement Program for Prostate Cancer (CLIPP) is associated with improvement in weight and components of metabolic syndrome in men exposed to androgen deprivation therapy for prostate cancer. Prostate Cancer Prostatic Dis. 2021. https://doi.org/10.1038/s41391-02100346-z. [Epub ahead of print].

10. Wilson RL, Newton RU, Taaffe DR, Hart NH, Lyons-Wall P, Galvao DA. Weight Loss for Obese Prostate Cancer Patients on Androgen Deprivation Therapy. Med Sci Sports Exerc. 2021;53 (Mar):470-8.

11. Wilson RL, Shannon T, Calton E, Galvão DA, Taaffe DR, Hart $\mathrm{NH}$, et al. Efficacy of a weight loss program prior to robot assisted 
radical prostatectomy in overweight and obese men with prostate cancer. Surg Oncol. 2020;35:182-8.

12. Galvão DA, Newton RU, Chambers SK, Spry N, Joseph D, Gardiner RA, et al. Psychological distress in men with prostate cancer undertaking androgen deprivation therapy: modifying effects of exercise from a year-long randomized controlled trial. Prostate Cancer Prostatic Dis. 2021. https://doi.org/10.1038/ s41391-021-00327-2. 